\title{
Improved Variable Step Size Least Mean Square Algorithm for Pipeline Noise
}

\author{
Xiaohui Zhang $\mathbb{D}^{D}$, Songnan Yang $\mathbb{D}^{D}$, Yuanyuan Liu $\mathbb{D}$, and Wei Zhao \\ Department of Information and Control Engineering, Xi'an University of Technology, Xi'an 710048, China \\ Correspondence should be addressed to Xiaohui Zhang; xhzhang@xaut.edu.cn
}

Received 9 December 2021; Revised 28 December 2021; Accepted 30 December 2021; Published 9 February 2022

Academic Editor: Tongguang $\mathrm{Ni}$

Copyright ( $\odot 2022$ Xiaohui Zhang et al. This is an open access article distributed under the Creative Commons Attribution License, which permits unrestricted use, distribution, and reproduction in any medium, provided the original work is properly cited.

\begin{abstract}
In this study, we employ the active noise control (ANC) method to eliminate the low-frequency part of the noise generated by the rotation of the axial fan in heating, ventilation, and air-conditioning (HVAC) pipelines. Because the traditional variable step size least mean square (VSS-LMS) algorithm has poor tracking performance, we propose a variable step size filtered-X least mean square (FXLMS) algorithm based on the arctangent function to improve the adaptive filtering method of the convergence speed and noise cancellation effect. The step size of the proposed algorithm can be adjusted according to the error. When the error signal is significant, a larger step can be obtained, and when the error is small, the step size smoothness of the algorithm can be optimized. Compared with the traditional VSS-LMS algorithm, the convergence speed of the proposed algorithm is increased by $29 \%$, the noise reduction effect is enhanced by $19 \%$, and the mean square error (MSE) is reduced by $23 \%(0.0084)$. In addition, we developed a hardware experimental platform based on noise characteristics. In the noise reduction test using a GB/T 5836.2-06 standard PVC pipeline, the system reduced the noise by $12-17 \mathrm{~dB}$.
\end{abstract}

\section{Introduction}

Noise has become one of the three significant pollutants that cannot be ignored globally due to the development of modern industry and the constant improvement of people's desires for a higher quality of life. In heating, ventilation, and air-conditioning (HVAC) systems [1], the noise generated during using compressors and axial flow fans is transmitted indoors through air pipelines, severely affecting people's production and life [2]. National laws and regulations have become more stringent regarding noise control requirements that affect public health. Research on various methods of reducing pipeline noise is significant for residential buildings, shopping malls, office buildings, factory workshops, and other comprehensive places greatly affected by noise [3]. Traditional noise control techniques adopt porous material sound absorption or baffle sound insulation to reduce pipe noise. Sound absorption and insulation only reduce mid and high-frequency noise but not lowfrequency noise [4]. Active noise control (ANC) technology can be widely used in noisy environments because of its effectiveness in low-frequency noise control.
In 1980, Morgan [5] proposed the filtered-X least mean square (FXLMS) method based on the least mean square (LMS) algorithm. In 2015, Ardekani et al. [6] modified the instantaneous gradient estimate by introducing the secondary path (SP) model to employ the FXLMS algorithm in ANC systems. In 2015, Zhou et al. [7] proposed a novel variable step size strategy for adaptive filtering algorithms, which depends on the sigmoid variable step size LMS (SVSLMS) algorithm. Because the change in step size is not smooth enough, the algorithm generates larger MSEs. In 2016, Wang et al. [8] proposed a sigmoid function called the antiinterference variable step size LMS (AVSS-LMS) adaptive filtering algorithm. In 2019, Salman et al. [9] proposed adding $l_{0}$ or $l_{1}$-norm penalty term to the cost function of the traditional LMS algorithm. Salman used the arctangent and sign functions to constrain weights in the iterative process of the filter weights to increase the sparsity of the LMS algorithm and further improve its convergence in impulse noise environments. In 2020, Bershad et al. [10] developed a new variable step size NLMS based on switched piecewise functions. Although the convergence speed is 
improved using the characteristics of each stage, at the beginning of the iteration, the algorithm still needs to set a fixed step size, which reduces the weakening effect of the sensor and environmental noise on the convergence performance of the algorithm. The improved method can reduce the steady-state error of convergence, but the improvement of convergence speed is limited. Since Salman's method is implemented by adding a penalty to the cost function, the adjustment of weights is delayed, and the penalty function is independent of the size of the error. Although the improved method is highly stable, the convergence effect is not ideal.

In this study, we suggested an optimized step change strategy for the VSS-LMS algorithm based on the ideas and research methodologies of Wang et al. [8], Salman et al. [9], and Bershad et al. [10]. We propose a variable step size FXLMS algorithm based on the arctangent function. The improved algorithm uses an inverse tangent function to link the error to the step size for the complex transformation rule of the step size in the VSS algorithm, and the transformed step size will find the optimum within a stable convergence range and does not require any new information for prediction. This optimization process is fully autonomous and does not require any perceived intervention, and the noise in the pipeline can be judged adaptively and effectively eliminated. The optimized convergence speed allows the system to adapt faster to changes in the external environment. Better robustness indicates that the active noise reduction system using this algorithm can be applied in a more complex environment, making the method more widely applicable to noise reduction in HAVC pipelines. The proposed algorithm improves the convergence speed of ANC and ensures the ANC system's stability. The simulation proceeds such that the convergence speed of the algorithm can be increased by $29 \%$ compared with that of the Wang et al. [8], Salman et al. [9], and Bershad et al. [10]. VSS-LMS algorithms and the noise cancellation effect of at least $12 \mathrm{~dB}$ can be achieved in the ANC experiment of HVAC pipelines. Simulations and experimental investigations show that using the proposed algorithm for the low-frequency part of the noise generated by the axial fan in the pipeline has a better effect.

The rest of this study is organized as follows: Section 2 introduces the HVAC-ANC system and experimental setup employed; Section 3 presents the traditional FXLMS algorithm and analyzes the step change strategy of the VSS-LMS algorithm; Section 4 introduces the improved algorithm, and Section 5 examines the axial fan noise characteristics in HVAC pipeline; Section 6 presents the algorithm simulation and experimental results; Section 7 presents the conclusion of the study.

\section{HVAC-ANC System}

2.1. HVAC System. People's concerns about the health threats of $\mathrm{PM}_{2.5}, \mathrm{PM}_{1.0}$, and other particulate pollutants, allergens, formaldehyde, total volatile organic compounds (TVOCs), household odors, and gaseous contaminants in the surrounding air driving forces for developing indoor air treatment and purification markets. An HVAC system is a technology for home environment amenities [11, 12]. The HVAC system provides thermal comfort and acceptable indoor air quality, including room temperature control, oxygen supplementation, and moisture removal. Figure 1 shows an HVAC system mainly composed of axial fans, HVAC pipelines, air filters, heating, and cooling units.

There are many noise sources in the pipeline, but their noise characteristics also have certain similarities because different systems have similar structures [13]. We analyze the main noise sources in the axial fan of an HVAC system, and some useful information can be provided for the design of an ANC system. Figure 2 shows the feedforward ANC system in an air pipeline. The reference microphone collects the noise sound wave $P 1$, and the controller outputs a control signal to the secondary speaker to generate an antinoise sound wave $P 2$. Then, the error microphone receives the synthesized sound waves $P 1$ and $P 2$ and feeds them back to the controller. From the combined sound energy density, when the phase difference between $P 1$ and $P 2$ is $180^{\circ}$, the combined sound energy density is the smallest. When the amplitudes of the sound waves $P 1$ and $P 2$ are equal, the energy of the sound wave reaching the error microphone is zero, achieving the purpose of active noise reduction.

\subsection{Simulation and Experimental Setup. MATLAB 2019 and} COMSOL Multiphysics 5.5 are used to simulate the algorithm. The input signal is Gaussian white noise plus sinusoidal at the frequency of $50 \mathrm{~Hz}$. The sample number is set as $n$, and the expected signal is a single frequency sinusoidal signal, as shown in Figure 3. Five comparison algorithms, including the proposed, fixed step size FXLMS, piecewise LMS, AVSS-LMS, and Salman algorithm, are used for comparison. The simulation experiment compares the convergence speed, MSE, and noise cancellation effect of the five algorithms. It is obvious from Figure 3 that the proposed algorithm can eliminate the random noise loaded in the sinusoidal signal, and the recovered signal has a good effect.

The experimental computer platform is shown in Figure 4. A polyvinyl chloride (PVC) pipeline following GB/ T5836.2-06 standard was used in the experiment. The pipe is $820 \mathrm{~mm} \times 110 \mathrm{~mm} \times 110 \mathrm{~mm}$ in size, and the size of the tee connection is $110 \mathrm{~mm}$. The test system uses a Yamaha-UR44 sound card, two Superlux-ECM999 standard acoustic measurement microphones, and two Philips monitor speakers. We use one monitoring speaker placed at the end of the pipe to generate test noise and the other speaker placed at the pipe tee to generate the cancellation sound waves. The reference microphone is located near the noise source during the test, and the error microphone is located behind the cancellation speaker. The position of the microphone will have a certain impact on the noise cancellation effect. It can have a good effect only after the Gaussian white noise is used for modeling. In practical use, once the modeling is completed, the position between the microphone and the speaker cannot change; otherwise, the system will be unstable. 


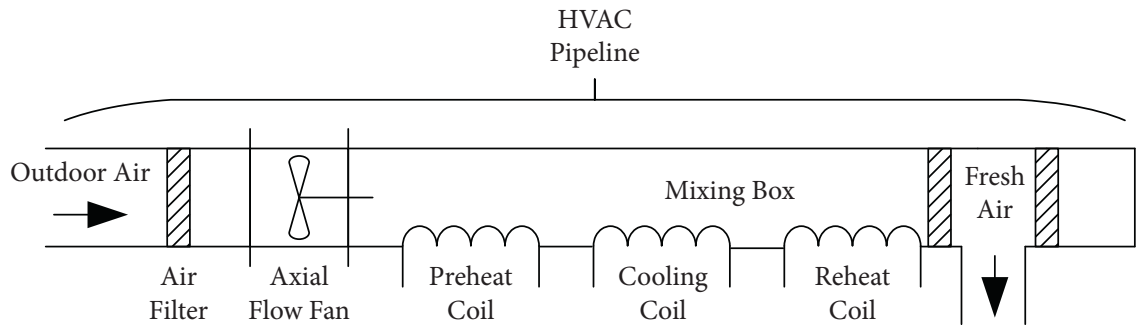

Figure 1: Schematic diagram of an HVAC system.

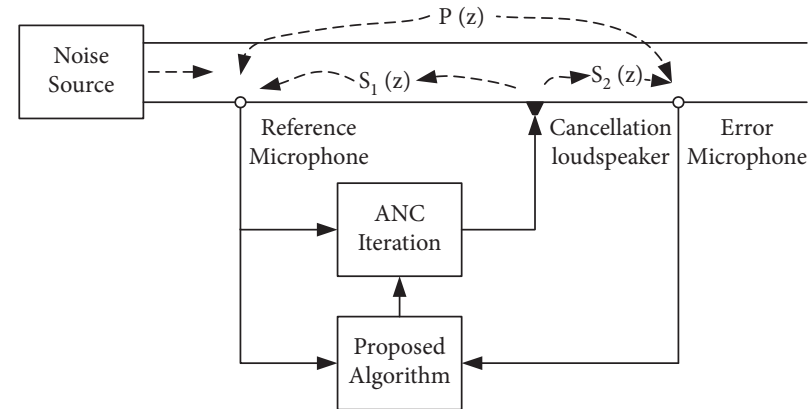

Figure 2: Feedforward ANC system in an HVAC pipeline.
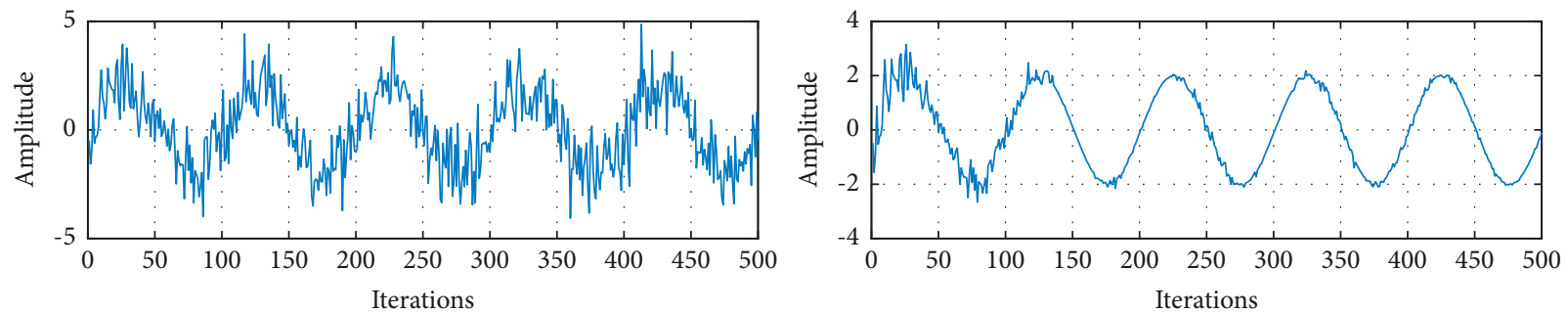

_ Origin Signal

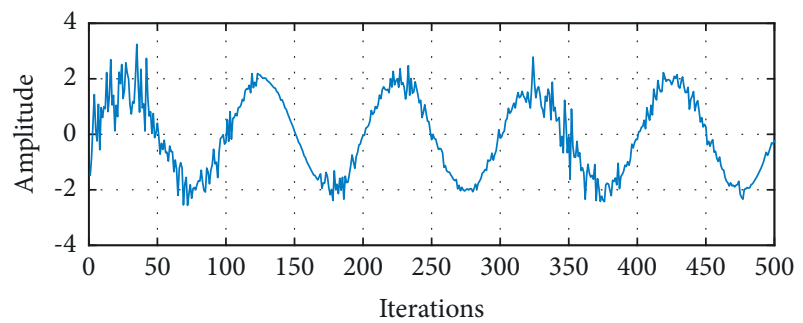

— Proposed

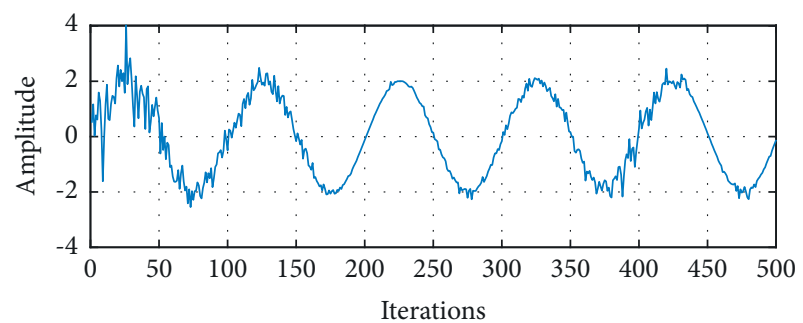

— AVSS-LMS

_ Fixed-Step-FxLMS
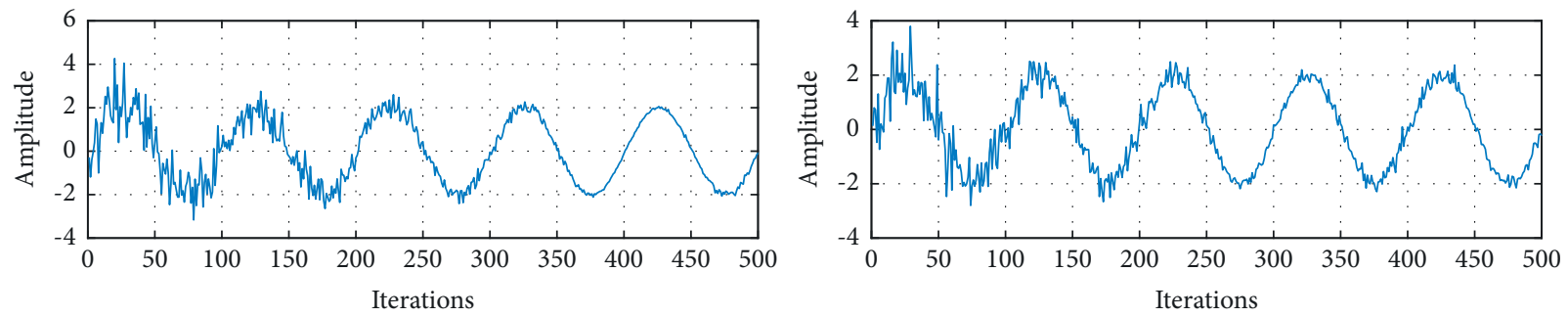

_ Piecewise LMS

— Salman

FIgURE 3: Comparison of five noise reduction methods. 


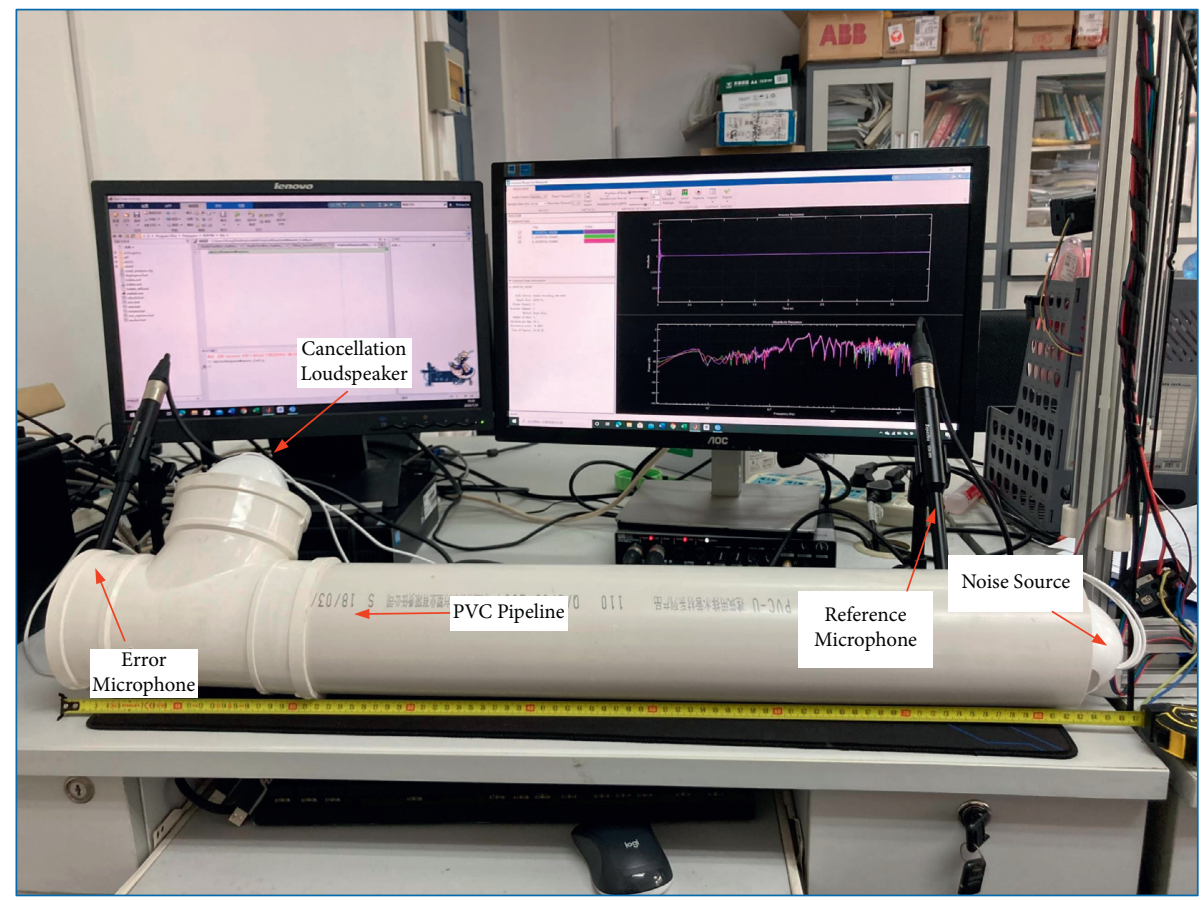

FIGURE 4: The experimental platform of a pipeline noise control.

\section{Active Noise Control Methods}

3.1. Fixed Step Size FXLMS Algorithm. The FXLMS algorithm modifies the error gradient estimation; hence, it is always used in pipeline ANC systems. Figure 5 shows a block diagram of the ANC system based on the FXLMS algorithm for air pipeline.

We can see in Figure 5 that $\mathbf{d}(n)$ denotes the desired signal, $\mathbf{x}(n)$ denotes the input signal, $\mathbf{u}(n)$ denotes the output signal, $\mathbf{e}(n)$ denotes the error between $\mathbf{y}(n)$ and $\mathbf{d}(n)$, and $\mathbf{w}(z)$ denotes the weight coefficient of the filter. The FXLMS algorithm employs the Wiener filter [14]. The Wiener filter is a linear optimal discrete filter. By calculating the autocorrelation matrix $R=E\left[\mathbf{u}(n) \mathbf{u}^{H}(n)\right]$ and crosscorrelation matrix $p=E[\mathbf{u}(n) \mathbf{d} *(n)]$, the filter with the smallest MSE can be obtained [15]. In order to obtain the optimal tap weight, the cost function is set as follows:

$$
\begin{aligned}
\xi= & E\left[d^{2}(n)\right]-w^{T} E[x(n) d(n)]-E\left[d(n) x^{T}(n)\right] w \\
& +w^{T} E\left[x(n) x^{T}(n)\right] w .
\end{aligned}
$$

The steps of the FXLMS algorithm are summarized as follows:

(a) The reference and error microphones collect the reference input signal $\mathbf{x}(n)$ and error signal $\mathbf{e}(n)$, respectively

(b) The output signal $\mathbf{u}(n)$ of the FIR filter with a length of $M$ is calculated, and the output signal $\mathbf{u}(n)$ drives the cancellation loudspeaker.

$$
u(n)=\sum_{i=0}^{M-1} w_{i}(n) x(n-i)
$$

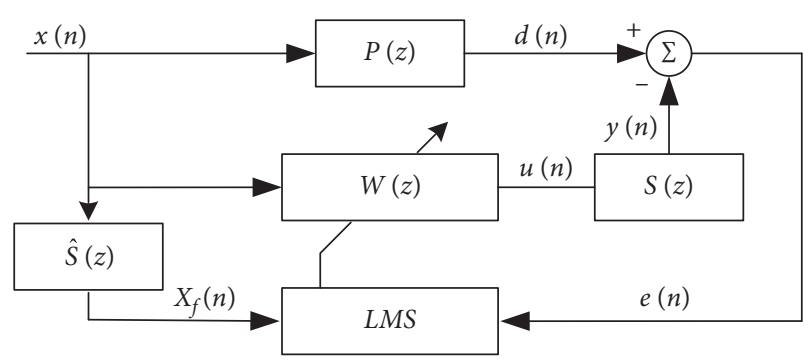

FIGURE 5: Feedforward FXLMS algorithm for an air pipeline.

(c) The filtered-X signal $\mathbf{x}_{f}(n)$ is calculated as the input signal of the LMS algorithm, where $\widehat{s}_{i}(n)$ is the $i^{\text {th }}$ impulse response coefficient of the SP $\widehat{S}(z)$ model at time $n$.

$$
x_{f}(n)=\sum_{i=0}^{M-1} \widehat{s}_{i}(n) x(n-i)
$$

(d) The iteration is repeated until the cost function $J(n)$ is minimized. Formula (5) indicates that the gradient of the cost function is obtained, and formula (6) indicates that the tap of the filter is optimized in the opposite direction of the gradient.

$$
J(n)=|e(n)|^{2} \approx\left(d(n)-\sum_{i=0}^{M-1} \widehat{s}_{i}(n) u(n-i)\right)^{2}
$$

$\nabla J_{i}(n)=\frac{\partial J(n)}{\partial w_{i}}$ 


$$
w_{i}(n+1)=w_{i}(n)-\frac{1}{2} \mu \nabla J_{i}(n) .
$$

(e) Using formula (6) to renew the weight coefficients of the LMS algorithm adaptive filter iteratively,

$$
W(n+1)=W(n)-2 \mu \mathbf{e}(n) \mathbf{x}_{\mathbf{f}}(n),
$$

where $\mu$ is the set convergence step size, and $e(n)$ is the error signal at the first iteration $e(0)=0$. The step is fixed in the original LMS algorithm [16]. However, the inherent limitation of the fixed $\mu$ needs an adjustment between fast convergence rate and limited misalignment. The step size range is expressed as the following formula to maintain the stability of the FXLMS algorithm.

$$
0<\mu<\frac{2}{\sum_{i=0}^{M-1}\left(x_{f}(n-i)\right)^{2}} .
$$

The FXLMS algorithm corrects the error gradient of the LMS algorithm by adding the estimated value of the SP, so that the LMS algorithm can converge stably.

The steps of SP modeling are summarized as follows:

(a) A white noise signal is set up by the white Gaussiannoise generator used as the input $S(z)$ to drive the cancellation loudspeaker, which is also used as the input signal $\widehat{S}(z)$ and the reference input signal of the LMS algorithm

(b) The error microphone collects the output signal of the cancellation loudspeaker as $y(n)$

(c) The output signal $\hat{y}(n)$ of the SP estimation model $\widehat{S}(z)$ is calculated as

$$
\hat{y}(n)=\sum_{i=0}^{M-1} \widehat{s}_{i}(n) u(n-i) .
$$

(d) The output variation between the actual path signal $y(n)$ and estimated path model $\hat{y}(n)$ is calculated as

$$
e(n)=y(n)-\hat{y}(n) .
$$

(e) The LMS algorithm updates the SP model of the adaptive filter as follows

$s_{i}(n+1)=s_{i}(n)+\mu e(n) \mu(n-i), \quad i=0,1,2, \ldots, M-1$.

(f) The above process is repeated until the error signal $e(n) \approx 0$, and the coefficient of $\widehat{S}(z)$ for the FXLMS algorithm is stored.

3.2. Variable Step Size LMS Algorithm. The VSS adaptive filtering algorithm uses a variable step factor $\mu(n)$. Changing the step factor can increase the convergence speed of the adaptive algorithm and reduce MSE. At the beginning of the algorithm or when $J(n)$ is large, a larger step size factor $\mu(n)$ is used to speed up the convergence. When the convergence phase or $J(n)$ is small, a smaller step factor is used to reduce MSE [17].

The AVSS-LMS algorithm based on the sigmoid function proposed by Wang et al. [8] is a very classic VSSLMS algorithm, and its step size factor $\mu(n)$ is given as

$$
\mu(n)=b\left(\frac{1}{1+\exp (-a E|e(n-1) e(n)|)}-0.5\right) .
$$

The parameter $a$ is used to handle the steepness of the sigmoid function and $b$ handles the value range of function. In this case, formula (7) can be expressed as

$$
W(n+1)=W(n)-2 \mu(n) x_{f}(n) .
$$

Compared with the step size $\mu$ of the FXLMS algorithm, the iterative step size $\mu(n)$ of the AVSS-LMS algorithm is variable. The initial stage of the algorithm convergence $\mu(n)$ has a high rate, implying a high convergence speed. In the stable stage, $\mu(n)$ takes a smaller value. When $b$ is $0.02,0.01$, or 0.005 and $a$ is $5,2,1$, or 0.5 , the step size of the AVSS-LMS algorithm varies with the error.

Figure 6 shows the step size change strategy of the AVSS-LMS algorithm. The AVSS-LMS algorithm solves the problem that the stability and convergence speed always contradict each other. However, the step size of the AVSSLMS change is not smooth enough when the error is limited, and the maximum step size of the function is limited by this problem. Here, the arctangent function is used to transform the error nonlinearly. The proposed algorithm can obtain a larger step size in the introductory stage. When it is close to stability, the step size changes smoothly to obtain rapid convergence speed and limited MSE.

\section{Improved Algorithm Using the Arctangent Function}

Although the principles of VSS-LMS algorithms are different, they all employ the basic adjustment strategy [18-21]. Mostly, VSS-LMS algorithms use a larger step size in the introductory moment of the iteration or when the error is large to increase the convergence rate. In the stable phase of the algorithm or when the error is limited [22], a limited step size factor is used to reduce MSE. This adjustment strategy is also employed here by introducing the arctangent function to construct the nonlinear accord between the error function and step size. The improved method adds three adjustable parameters for different noises. The improved step change coefficient is given as

$$
\mu(n)=b\left(\frac{3}{\left(1+\exp (-a|\arctan (e(n))|)^{c}\right)}-1\right),
$$

where $\mu(n)$ represents the step value obtained when the error at the time $n$ is $e(n)$, and $a, b$, and $c$ are the user-defined parameters and constants. The arctangent function is used for three reasons; it is nonlinear, bounded, and continuous. These three points can ensure the convergence and stability of the function. 


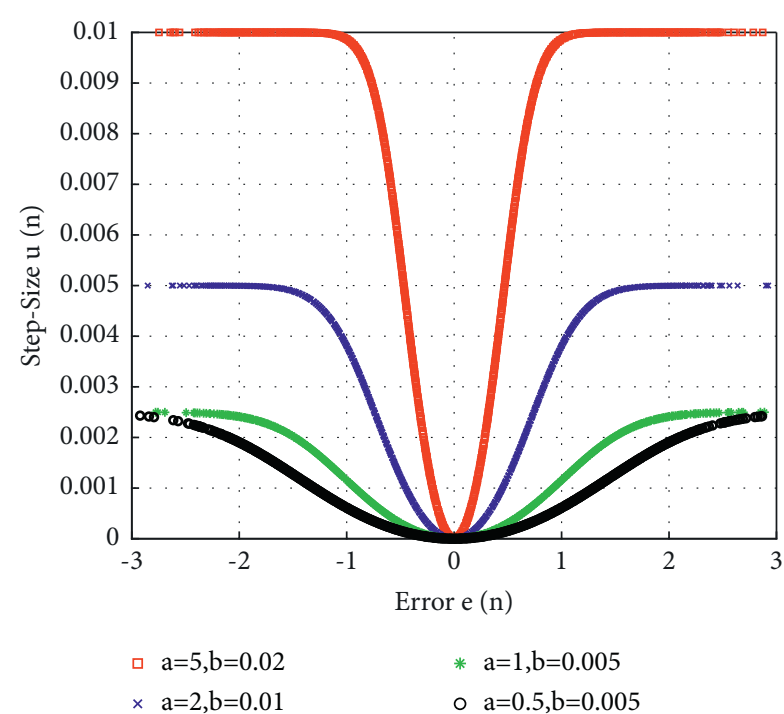

FIgURE 6: Variation of the step size of the AVSS-LMS algorithm with error.

4.1. Effect of Step Size on the Algorithm. We conclude from formula (15) that increasing the step size factor can reduce the learning time and converge a little time. However, the small step length will boost the number of iterations [23], thus reducing the convergence acceleration of the algorithm.

$$
\tau_{m s e, \min } \approx \frac{1}{2 \mu(n) \lambda_{\min }},
$$

where $\tau_{m s e, \min }$ represents the smallest eigenvalue of the autocorrelation matrix of the input signal and $\lambda_{\text {min }}$ represents the minimum MSE that can be obtained by the algorithm. Therefore, to solve the problem of the stability and convergence speed always contradicting each other [24] when the fixed step size adaptive filtering algorithm is tested to the active control of pipeline noise, the strategy of changing the step size is employed. Formula (16) shows that the steady-state error is directly proportional to the step size. As the step size increases, the steady-state error increases [25].

$$
\varepsilon=\frac{\mu(n)}{2} \sum_{k=1}^{M} \lambda_{k}
$$

where $\lambda_{k}$ is the eigenvalue of the autocorrelation matrix $\mathbf{u}(n)$. Formulas (15) and (16) show a contradictory relationship between the convergence rate and stability.

4.2. Effect of Parameter $a$ on the Algorithm. Parameters $b$ and $c$ are fixed to investigate the effects of parameter $a$ and step size $\mu$ of the proposed algorithm. When $b=0.03, c=2$, and $a=5,2,1$, and 0.5 (Figure 7), the step size $\mu$ of the improved algorithm varies with the error $e(n)$. With an increase in $a$, the step variation curve gradually becomes steep. It means that a larger parameter $a$ can make the algorithm more sensitive to error, but when $a$ increases to a certain extent, the maximum step size of the algorithm will not continue to increase.

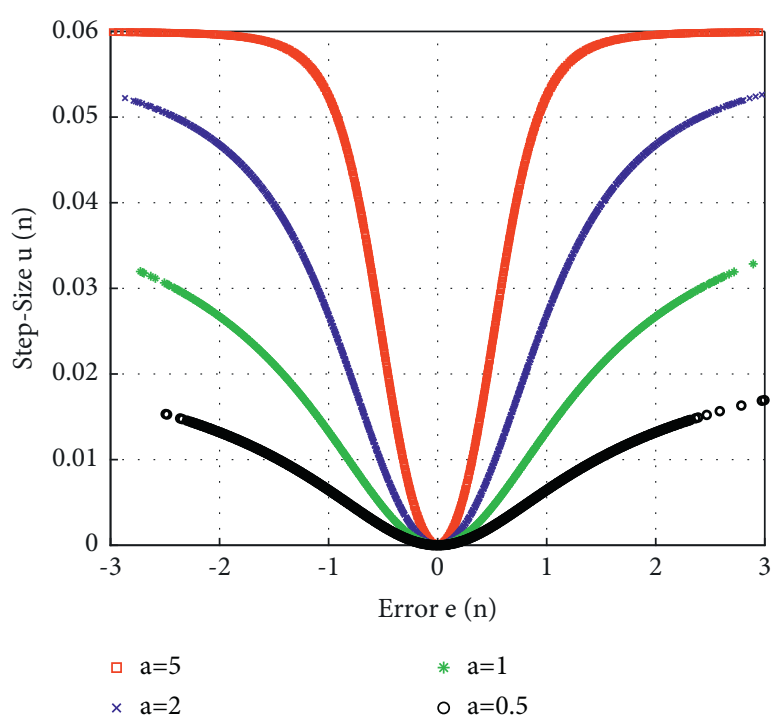

FIgURE 7: Variation of the step size of the proposed algorithm with a.

Figure 8 shows that as a gradually increases, the convergence speed increases rapidly. The convergence speed of the algorithm is influenced by $a$. As the value of $a$ increases, the convergence speed becomes brisker, simultaneous bottom becomes sharper, and MSE increases accordingly. We can see from formula (16) that this is caused by the increase of step size. Therefore, blindly increasing parameter $a$ is not a good strategy to improve the algorithm. It can be seen from Figure 8 that when $a>2$, the increase in the convergence speed of the algorithm is not so obvious, so the value of parameter $a$ cannot be set too large.

4.3. Effect of Parameter $b$ on the Algorithm. The influence of parameter $b$ on the step size $\mu$ of the improved algorithm is studied by fixing the parameters $a$ and $c$. When the parameters $a=1, c=2$, and $b=0.005,0.01,0.03$, and 0.1 , the step size varies with the error, as shown in Figure 9. Parameter $b$ directly affects the maximum step size of the algorithm. As $b$ increases, the upper limit of the maximum step size increases, but very high values of $b$ may increase the MSE.

As shown in Figure 10, at a smaller $b$, the convergence acceleration of the algorithm is greatly reduced. Therefore, to ensure the high convergence acceleration, stable convergence of the algorithm $b$ should be adjusted according to the stable convergence conditions shown in the following formula:

$$
0<\mu<\frac{2}{\sum_{i=0}^{M-1}\left(x_{f}(n-i)\right)^{2}}
$$

The algorithm will not converge when the variable step size exceeds the upper limit. Therefore, the reciprocal of the input signal power is usually used to determine the upper limit of the step size. 


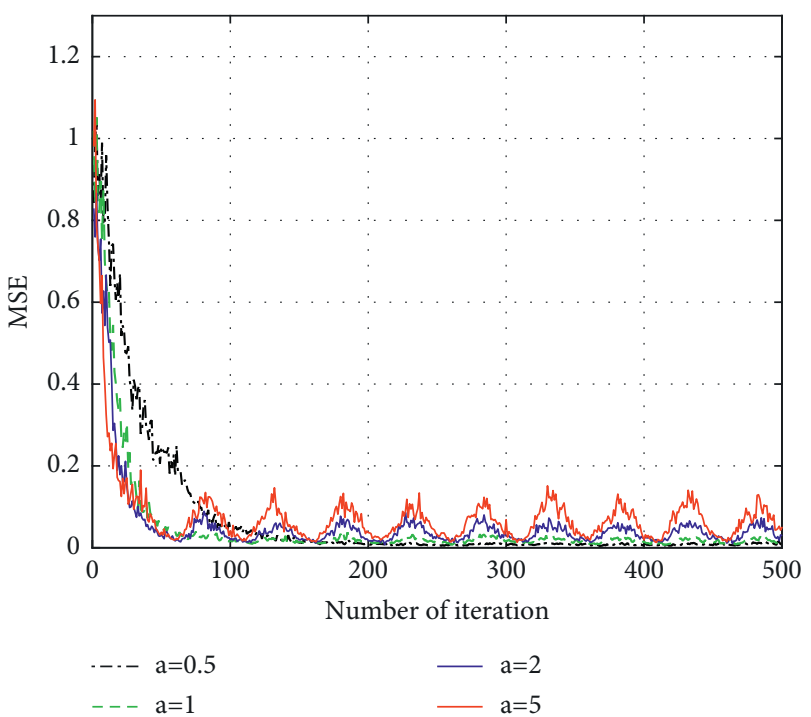

Figure 8: MSE of the proposed algorithm at different $a$.

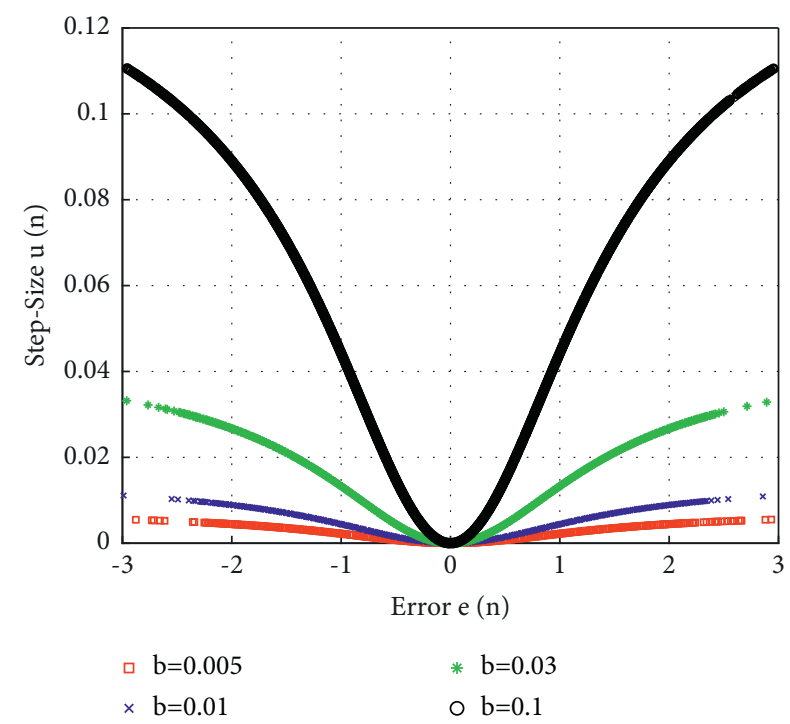

FIGURE 9: Variation of the step size of the proposed algorithm with $b$.

4.4. Effect of Parameter $c$ on the Algorithm. The influence of $c$ on the algorithm is studied by fixing $a$ and $c$. When $a, b$, and $c$ are $0.5,1,2$, and 4 , the step size varies with the error, as shown in Figure 11, and we can see that $c$ directly affects the smoothness of the bottom of the function; the larger the value of $c$, the smoother bottom of the function. Without affecting the steady-state error, increasing the value of $c$ increases the convergence speed of the algorithm to a certain extent without affecting the stability of the function. Since the value $a$ directly affects the way of a step change, according to the variable step strategy, the value $c$ should be greater than 1. Because exponential growth directly increases the amount of calculation (Figure 12), the convergence speed of the algorithm is not significantly increased only by

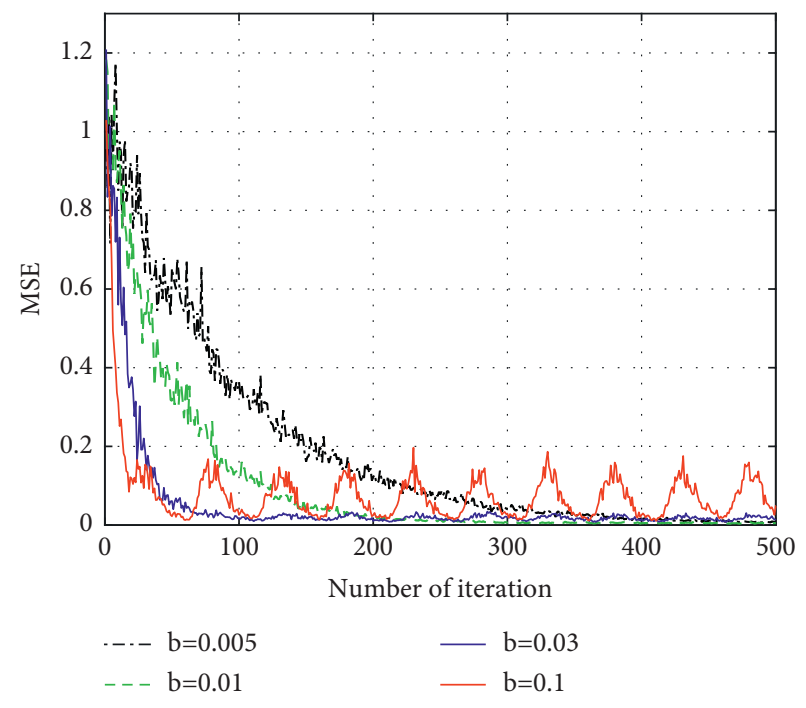

FIgURE 10: MSE of the proposed algorithm at different $b$.

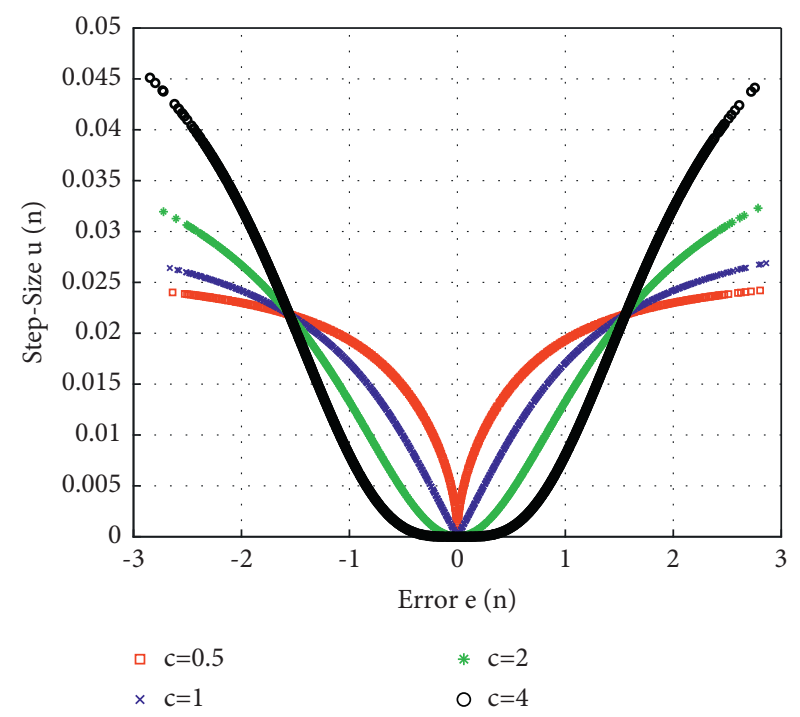

FIGURE 11: Variation of the step size of the proposed algorithm with c.

adjusting the value of $c$. Therefore, the value of $c$ needs to be adjusted after adjusting that of $a$ and $b$.

4.5. Robustness of the Algorithm. Random noise has a severe impact on the stability of the adaptive algorithm [26]. A random addition is needed for a robust solution for the adaptive filter due to the inevitable noise in the environment, such as measurement noise and environmental modeling errors [27]. Therefore, it is necessary to introduce a random additive interference signal $v(n)$. The expected signal $d(n)$ is expressed as

$$
d(n)=w_{o}^{H}(n) u(n)+v(n)
$$




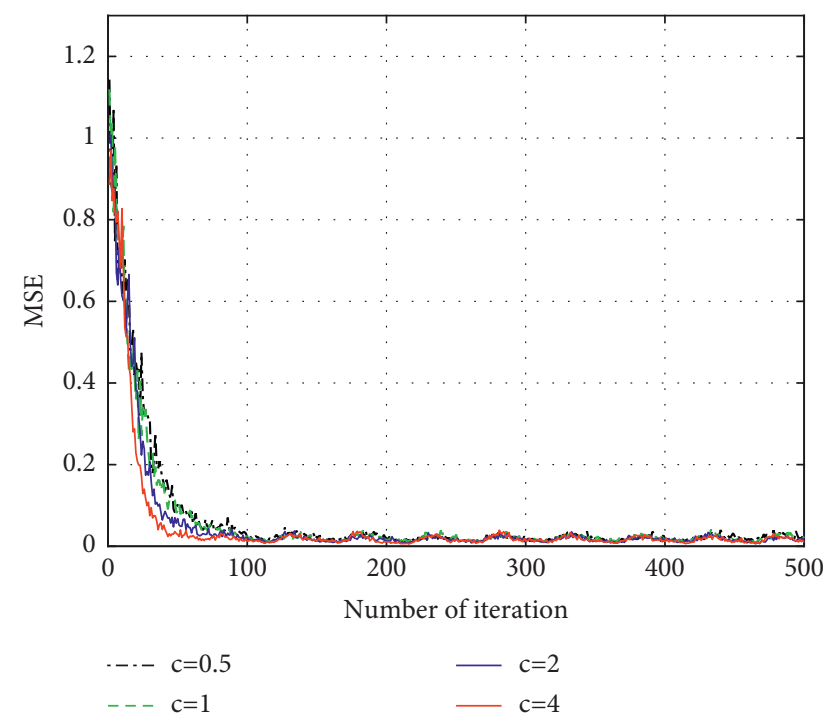

FIGURE 12: MSE of the proposed algorithm at different $c$.

where $w_{o}(n)$ is the optimal tap weight vector of the Wiener filter. However, because the environment of the system is unknown, suppose that $w_{o}(n)$ is estimated as $w(n)$, the expected signal can also be expressed by formula (25).

$$
d(n)=w(n) u(n)+e(n)
$$

Since the recursive estimation of $w(n)$ is strictly causal, in the adaptation cycle $n$, updating $w(n-1)$ to $w(n)$ depends only on the current input vector $u(n)$, and the weighted error vector $\widetilde{w}(n)$ can be defined as

$$
\widetilde{w}(n)=w_{o}(n)-w(n) .
$$

As the measurable error signal relevant to the weighted error vector $w(n)$, the error without interference is given by

$$
\begin{aligned}
\xi_{u}(n) & =e(n)-v(n) \\
& =\left[w_{o}(n)-w(n)\right]^{H} u(n) \\
& =\widetilde{w}(n) u(n),
\end{aligned}
$$

where $v(n)$ is the random additive interference signal, $e(n)$ is the error signal, and the cause of $\xi_{u}(n)$ can be known from formula (20) as the deviation between the optimal solution $w_{o}(n)$ of the Wiener filter and the actual filter $w(n)$.

We calculate the arbitrary estimator $H^{\infty}$, and it is necessary to calculate the worst-case energy gain from the interference [28] to the noninterference estimation error in formula (21). To obtain the bounds of the energy gain, we can obtain the following formula from the Cauchy-Schwartz theorem.

$$
[\widetilde{w}(n) u(n)]^{2} \leq\|\widetilde{w}(n)\|^{2}\|u(n)\|^{2}
$$

Therefore, by taking formula (21) squared at both ends and based on the inequality shown in formula (22), the following conclusions can be drawn:

$$
\left|\xi_{u}(n)\right|^{2} \leq\|\widetilde{w}(n)\|^{2}\|u(n)\|^{2} .
$$

Add interference $v(n)$ to the right of formula (23), and since $\mu(n)$ is always positive and less than 1 , we can obtain the inequality shown in formula.

$$
\left|\xi_{n}(n)\right|^{2} \leq \mu^{-1}(n)\|\widetilde{w}(n)\|^{2}+|v(n)|^{2} .
$$

When the algorithm converges, it is necessary to ensure that $\widetilde{w}(n+1) \leq \widetilde{w}(n)$, so the inequality shown in formula (25) can be derived from formula (24):

$$
\mu^{-1}(n)\|\widetilde{w}(n+1)\|^{2}+\left|\xi_{\mu}(n)\right|^{2} \leq \mu^{-1}(n)\|\widetilde{w}(n)\|^{2}+|v(n)|^{2} .
$$

Since the estimated value of the tap $\widetilde{w}(n)$ is monotonically decreasing, we can obtain the inequality shown in the following formula:

$$
\mu^{-1}(n)\|\widetilde{w}(n)\|^{2}+\sum_{n=0}^{N}\left|\xi_{u}(n)\right|^{2} \leq \mu^{-1}(n)\|\widetilde{w}(0)\|^{2}+\sum_{n=0}^{N}|v(n)|^{2} .
$$

If formula (23) is brought into formula (26), there must be an inequality shown in formula (27), which shows that the proposed method will have good robustness when the following conditions are met.

$$
\sum_{n=0}^{N}\left|\xi_{u}(n)\right|^{2} \leq \mu^{-1}\|\widetilde{w}(0)\|^{2}+\sum_{n=0}^{N}|v(n)|^{2}
$$

The right side of formula (27) can be divided to the left, and the symbol $\gamma^{2}$ represents the maximum energy gain.

$$
\gamma^{2}(\mu(n))=\operatorname{SUP}_{w, v \in H^{2}} \frac{\sum_{n=0}^{N}\left|\xi_{u}(n)\right|^{2}}{\mu^{-1}\|\widetilde{w}(0)\|^{2}+\sum_{n=0}^{N}|v(n)|^{2}},
$$

where superscript 2 represents the second norm, and sup means the supermum upper bound. Assuming the specific interference sequence is $v(n)=-\xi_{u}(n)$, so formula (28) can be expressed as

$$
\gamma^{2}(\mu(n))=\frac{\sum_{n=0}^{N}\left|\xi_{u}(n)\right|^{2}}{\mu^{-1}\|\widetilde{w}(0)\|^{2}+\sum_{n=0}^{N}\left|\xi_{u}(n)\right|^{2}} .
$$

Therefore, when the power of the input signal $u(n)$ is limited, it is $\lim \sum_{n=0}^{N}\|u(n)\|^{2}<\infty$. For any given normal number $\Delta$, a parameter vector $w(n)$, and an integer $N$, formula (21) can be expressed as

$$
\begin{aligned}
\sum_{n=0}^{N}\left|\xi_{u}(n)\right|^{2} & =\sum_{n=0}^{N}\left|\left(w_{o}(n)-w(n)\right)^{H} u(n)\right|^{2} \\
& \geq \frac{1}{\Delta \mu(n)}\left\|w_{o}(n)-w(0)\right\|^{2} \\
& =\frac{1}{\Delta \mu}\|\widetilde{w}(0)\|^{2} .
\end{aligned}
$$

By eliminating the common term of formula (29) and keeping the bounds of $\gamma^{2}$, we can get the result 


$$
\frac{1}{1+\Delta} \leq \gamma^{2} \leq 1
$$

Formula (31) shows that as the constant $\Delta$ tends to 0 , the maximum energy of the improved algorithm will never exceed 1 . Therefore, the proposed algorithm is $H^{\infty}$ optimal, and the system has good stability [29].

\section{Characteristics of Pipeline Noise}

There are many noise sources in a pipeline. However, due to the similarity in the structure of different systems, their noise characteristics are also similar [30]. The rotating noise is the main source of pipeline noise, and its fundamental frequency can be determined by the number of fan blades and motor velocity $[31,32]$. The noise produced by the axial flow fan is more than $25 \mathrm{~dB}$ (Table 1). Here, we use the POPULAJD100 axial fan (Figure 13).

The noise generated by the axial fan can be calculated using the following formulas [33]:

$$
\begin{aligned}
\mathrm{PWL}= & 56 \lg V+10 \lg \left(p_{s} c_{m}^{0.6} L Z\right)-15.23 \\
V= & 2 \pi r\left(\frac{n}{60}\right) \\
p_{s}= & p_{i}+p_{d} \\
\mathrm{SPL}= & 56 \lg V+10 \lg \left(p_{s} c_{m}^{0.6} \mathrm{LZ}\right)-15.23 \\
& -20 \lg (4 \pi d)+10.99 .
\end{aligned}
$$

The static pressure $p_{i}=101325(\mathrm{~Pa})$, dynamic pressure $p_{d}=274(\mathrm{~Pa})$, motor speed $n=2450(\mathrm{rpm})$, average width of the fan blade $C_{m}=0.015(\mathrm{~m})$, leaf height $L=0.020(\mathrm{~m})$, and the number of leaves $Z=10 . d$ is the measured distance, and the constant number 15.23 is the empirical value. The simulation is performed according to formula (35). The noise of the axial fan is mainly produced by monopole, dipole, and quadrupole noise sources [34]. Monopole noise is mainly caused by a change in the gas volume [35], dipole noise by the fluctuation of the static pressure on the impeller surface, and quadrupole noise by viscous stress radiation [36]. Due to the low air velocity of the JD100 axial flow fan, the monopole noise source can be ignored, and in the case of a low Mach number, the quadrupole noise source can be ignored. Therefore, the aerodynamic noise of the JD100 axial fan is mainly a dipole noise source. Axial flow fans produce different noises under different working conditions, and their frequency, sound pressure, and speed are not linear. According to formula (35), the sound pressure spectrum distribution of JD100 axial flow fan at different distances from 0 to $2500 \mathrm{rpm}$ is studied. Figure 14 shows that the fundamental frequency and harmonics of the aerodynamic noise generated by the JD100 axial flow fan gradually increase with speed. The maximum frequency of noise is obtained at a speed of $2500 \mathrm{rpm}$. The main frequency of the rotating noise is below $4 \mathrm{kHz}$, which indicates medium and low-frequency noise. It is difficult to reduce this part of noise using the passive noise reduction method. Figure 15 shows that the higher the fan speed [37], the closer the measurement distance and the greater the noise obtained. At $2500 \mathrm{rpm}$, the noise generated by the JD100 axial flow fan is not less than $30 \mathrm{~dB}$ when the distance is $1 \mathrm{~m}$.

Noise mainly propagates through the pipe, and the shape, size, and wall material of the pipe affect sound wave propagation in the pipe [38]. To analyze this problem, first, a cylindrical coordinate system $O-r-\varphi-z$ is established for a circular pipe, as shown in Figure 16, assuming that noise is generated at $\mathrm{z}=0$ of the tube and the other end is infinitely extended. The wall of the pipe is assumed to be rigid. The acoustic field in the pipe varies in the range $r \in[0, a]$, $\varphi \in[0,2 \pi]$, and $z \in[0, \infty]$.

Assuming the relationship between the generator's sound pressure and time is simple and harmonic, the sound pressure can be expressed as

$$
\begin{aligned}
p(x, y, z, t) & =p(x, y, z) e^{j \omega t}, \\
\nabla^{2} p(x, y, z)+k^{2} p(x, y, z) & =0, \\
\nabla^{2} & =\frac{1}{r} \frac{\partial}{\partial r}\left(r \frac{\partial}{\partial r}\right)+\frac{1}{r^{2}} \frac{\partial^{2}}{\partial \varphi^{2}}+\frac{\partial^{2}}{\partial z^{2}},
\end{aligned}
$$

where $\nabla^{2}$ is the Laplacian of the three-dimensional cylindrical coordinate system, $k$ is the number of waves, and $c$ is the sound speed [39]. Then, the sound field in the rigidwalled cylindrical waveguide under excitation of the nonaxisymmetric sound source is given by

$$
\begin{aligned}
p(r, \varphi, z, t)= & e^{j \omega t} \sum_{n} \sum_{m} A_{n m} J_{n}\left(\frac{\beta_{n m}}{a} r\right) \cdot \cos \left(n \varphi+\varphi_{n}\right) \\
& e^{-j \sqrt{k^{2}-\left(\beta_{n m} / a\right)^{2} z}},
\end{aligned}
$$

where $A_{n m}$ is the constant determined by the boundary conditions, $n$ and $m$ are the circumferential and radial modal numbers, respectively, $a$ is the radius of the pipe, and $J_{n}(x)$ is the first Bessel function. The value of $\beta_{n m}$ can be obtained from Table 2.

On the cross-section of a circular tube, the normal mode wave of order $(n, m)$ determined at different values of $\beta_{n m}$ would result in different acoustic characteristics [40]. The sound pressure in a pipe is the superposition of the sound pressure components of each model. From formula (39), we can obtain an important property of sound wave propagation in a circular tube, that is, the existence of cutoff frequency $n$, as

$$
\begin{aligned}
\sqrt{k^{2}-\left(\frac{\beta_{n m}}{a}\right)^{2}} & <0, \\
f_{\text {cutoff }} & \geq \frac{\beta_{m n}}{2 \pi a} c, \\
c & =\sqrt{\Upsilon R T},
\end{aligned}
$$


TABle 1: Axial fan performance parameter table.

\begin{tabular}{|c|c|c|c|c|}
\hline \multirow{2}{*}{ Model (no.) } & \multicolumn{4}{|c|}{ Data parameter } \\
\hline & Power $(\mathrm{W})$ & Air volume $\left(\mathrm{m}^{3} / \mathrm{h}\right)$ & Pressure $(\mathrm{Pa})$ & Noise $(\mathrm{dB})$ \\
\hline JD100 & $28 / 25$ & $200 / 150$ & $180 / 130$ & $30 / 25$ \\
\hline JD150 & $50 / 40$ & $500 / 400$ & $300 / 250$ & $35 / 30$ \\
\hline JD200 & $70 / 60$ & $850 / 700$ & $350 / 280$ & $50 / 45$ \\
\hline JD250 & $170 / 130$ & $1500 / 1100$ & $550 / 400$ & $60 / 54$ \\
\hline JD315 & $320 / 230$ & $2300 / 1800$ & $700 / 500$ & $66 / 61$ \\
\hline
\end{tabular}

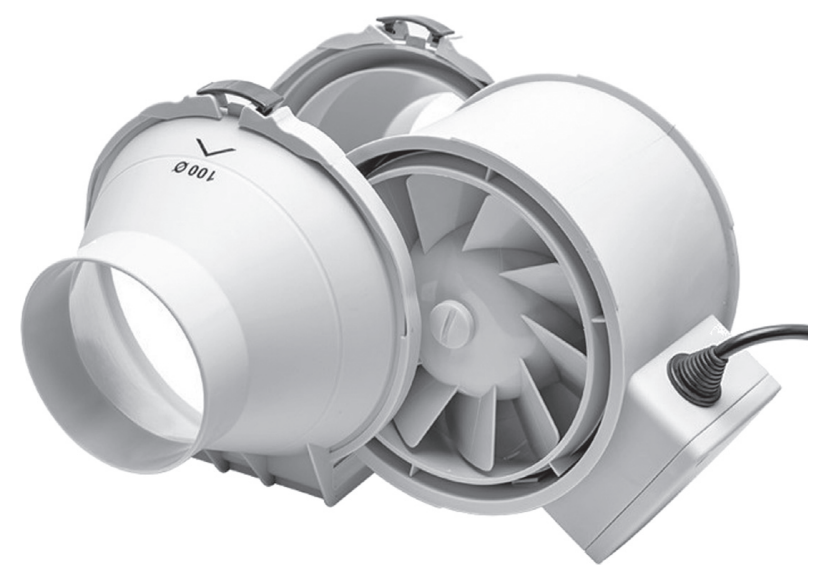

Figure 13: POPULA-JD100 axial fan.

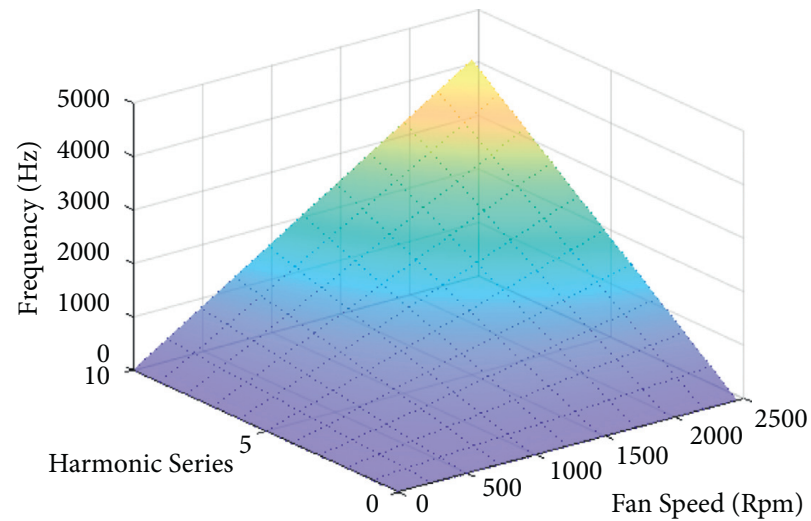

FIGURE 14: Relationship between speed and noise frequency of an axial fan.

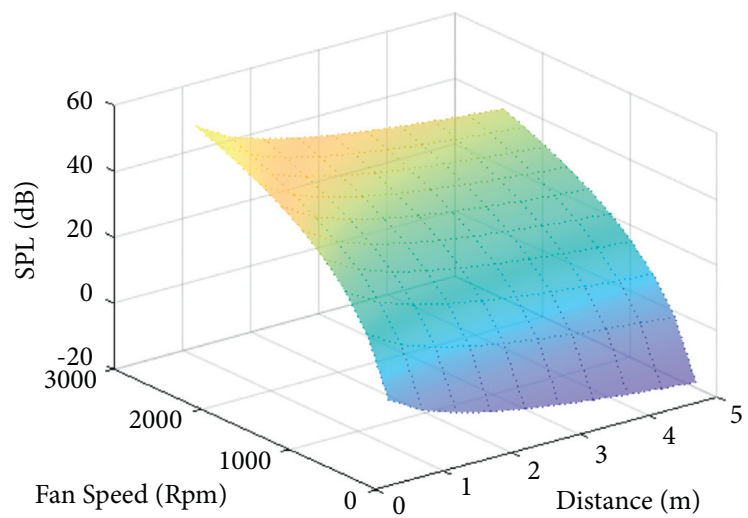

FIGURE 15: Relationship between noise and distance of an axial fan. 


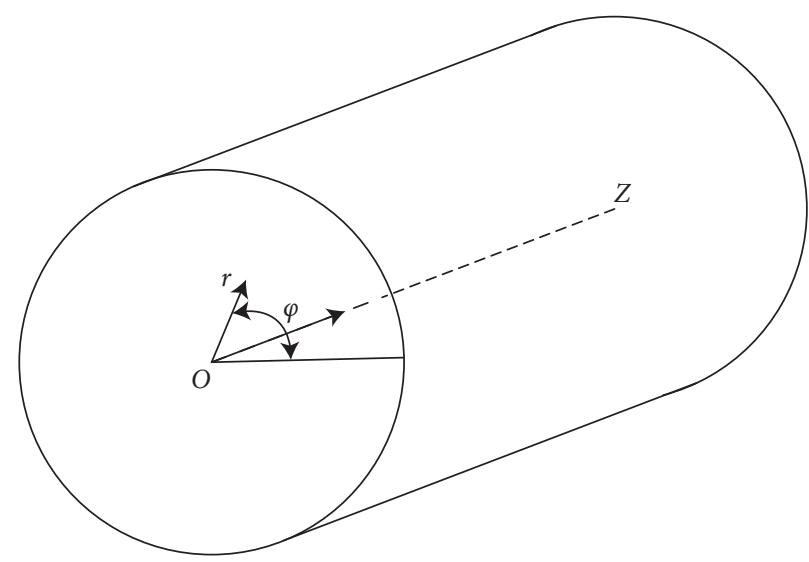

FIGURE 16: Circular pipe waveguide.

TABLE 2: $\beta_{n m}$ root table.

\begin{tabular}{|c|c|c|c|c|c|c|}
\hline$\beta_{n m}$ & $n=0$ & $n=1$ & $n=2$ & $n=3$ & $n=4$ & $n=5$ \\
\hline$m=0$ & 0 & 3.832 & 7.016 & 10.174 & 13.324 & 16.47 \\
\hline$m=1$ & 1.841 & 5.331 & 8.536 & 11.706 & 14.864 & 18.016 \\
\hline$m=2$ & 3.054 & 6.706 & 9.969 & 13.17 & 16.348 & 19.513 \\
\hline$m=3$ & 4.201 & 8.015 & 11.346 & 14.586 & 17.789 & 20.973 \\
\hline$m=4$ & 5.318 & 9.282 & 12.682 & 15.964 & 19.196 & 22.401 \\
\hline$m=5$ & 6.415 & 13.987 & 13.987 & 17.313 & 20.576 & 23.804 \\
\hline
\end{tabular}

where $\Upsilon$ is the specific heat ratio (for air $\Upsilon=1.4$ ), $\mathrm{T}$ is the thermodynamic temperature of the gas in $K$, and $R$ is the gas constant (usually $R=287 \mathrm{~J}(\mathrm{~kg} \cdot \mathrm{K})$ ). If there is no pressure change during a specific use, only the temperature is usually considered. In the pipeline, the sound wave frequency is smaller than the cutoff frequency that can propagate by formula (39), and the sound wave frequency larger than the cutoff frequency will decay exponentially. The noise higher than the cutoff frequency does not need ANC [41]. The frequency of the pipeline is a result of the superposition of multiple groups of $\beta_{n m}$ values that can be obtained from Table 2. In the finite element analysis of the pipeline [42], white noise is used to excite the simulation model, and the simulation model has the attenuation effect of excitation sound waves at different frequencies $(350-3600 \mathrm{~Hz})$, as shown in Figure 17.

The simulation of the sound wave transmission aspect of the pipeline shows that, for the HVAC-ANC system, highfrequency noise generated by the axial fan is quickly attenuated in the pipeline. Therefore, the ANC system can only handle frequencies below $4 \mathrm{kHz}$ at the end of the pipeline. Low-frequency noise can reduce the acquisition bandwidth of ADC [43], reduce the number of iterations of the ANC system, and improve the noise cancellation effect of the algorithm.

\section{Experimental Results and Discussion}

The proposed algorithm is compared with the fixed step size FXLMS, piecewise LMS, AVSS-LMS, and Salman algorithms in the simulation. Table 3 provides the parameter setting. The test input signal is composed of Gaussian white noise and sinusoidal signal. The system has 10000 iterations, and the signal noise ratio (SNR) is $20 \mathrm{~dB}$.

Figure 18 shows the step sizes of the five algorithms very differently according to the error signal size variation trend. The proposed algorithm can obtain the maximum step size when the error is large. According to formula (15), the larger the step size, the higher the convergence speed of the algorithm. Therefore, the algorithm is characterized by high convergence speed when the error signal is large, and the step size obtained from the piecewise LMS algorithm occurs second. When the error signal is small, the step change of the proposed algorithm is smoother than that of the AVSS-LMS and piecewise LMS algorithms. Formula (16) shows that the steady-state error obtained with a smaller step size becomes smaller as it approaches stability. Therefore, compared with the AVSS-LMS and piecewise LMS algorithms, the proposed method has less MSE. Formula (30) shows that the proposed method has a high convergence speed and reduced MSE. It also has good antiinterference ability and can converge stably.

Figure 19 shows that the five algorithms can converge and filter out the noise, but their convergence speed and MSE are different; the speed and MSE of the proposed algorithm are better than those of the other four algorithms. Figure 19 shows that, among other algorithms, the performance of the piecewise LMS algorithm is closest to the convergence performance of the proposed algorithm. The proposed and piecewise LMS algorithms have good theoretical tracking performance on noise, but the piecewise points of the piecewise LMS algorithm affect the results of the algorithm. In order to improve the performance of the piecewise LMS algorithm, much time and energy are needed 

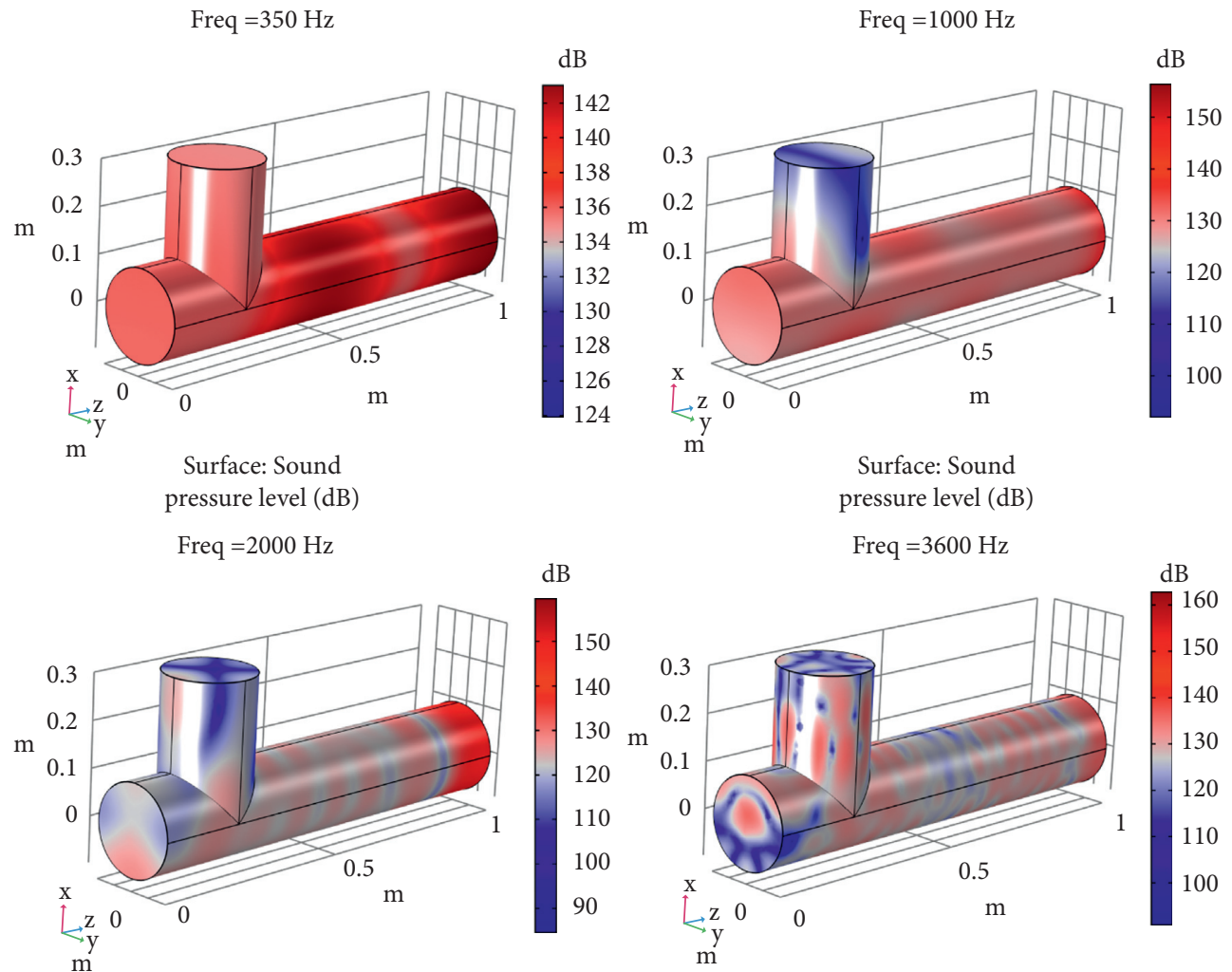

Surface: Sound
pressure level $(\mathrm{dB})$

Surface: Sound
pressure level $(\mathrm{dB})$

FIGURE 17: Sound wave transmission simulation in a PVC pipeline.

TAвLE 3: Simulation parameters for the proposed algorithm and other algorithm.

\begin{tabular}{|c|c|c|}
\hline Algorithm & Variable step & Parameter \\
\hline AVSS-LMS & $\mu(n)=b(1 / 1+\exp (-a E|e(n-1) e(n)|)-0.5)$ & $a=0.97, b=0.03$ \\
\hline Fixed step FXLMS & $\mu$ & $\mu=0.01$ \\
\hline Piecewise LMS & $\left\{\begin{array}{l}\mu=k, 0 \leq n \leq \text { sample length } / 5 \\
\mu(n)=b\left(1-\exp \left(-a *\left|e^{2}(n)\right|\right)\right), 0.001<\left|e^{2}(n)\right| \leq 0.01 \\
\mu(n)=b(-1+\sec (a *|e(n)|)), 0 \leq\left|e^{2}(n)\right| \leq 0.001\end{array}\right.$ & $k=0.03, a=0.97, b=0.03$ \\
\hline Salman & $\mu(n)=\lambda\left(\operatorname{sgn}(w(n)) /\left(1+b|w(n)|+b^{2}|w(n)|^{2}\right)\right)$ & $\lambda=0.02, a=0.97, b=0.03$ \\
\hline Proposed & $\mu(n)=b\left(3 /\left(1+\exp (-a|\arctan (e(n))|)^{c}\right)-1\right)$ & $a=0.97, b=0.03, c=4$ \\
\hline
\end{tabular}

to fine-tune the parameters. Considering MSE, the Salman algorithm has a low MSE after stability. The proposed method can have MSE as low as that of the Salman algorithm, but its convergence speed is much higher than that of other algorithms. Notably, the MSE of the proposed algorithm shows a further downward trend.

Table 3 provides that the Salman algorithm is also a variable step size algorithm, but Figure 18 shows that the variable step size strategy of the Salman algorithm is not causally related to the error signal. The Salman algorithm uses a penalty weight to adjust the step size. It makes the weight lag, so it does not have a large step at the beginning of iterations. Its convergence speed is much lower than the AVSS-LMS and piecewise LMS algorithms (Figure 19). However, it can obtain smaller MSE than the AVSS-LMS and piecewise LMS algorithms when the number of iterations increases and it converges stably. The Salman algorithm can obtain the same noise cancellation effect as the proposed algorithm when it is employed in the processing of HVAC pipeline noise. Still, its ability to track abrupt noise is not as strong as that of the proposed algorithm.

ANC-OFF in Figure 20 is the noise collected by the microphone at the outlet of the PVC pipe, and it can be seen that part of the noise generated by the axial fan, part of the rotating noise has a frequency, which can be deduced from formula (32), while the other part belongs to the random noise, and this part of the noise belongs to the more difficult to eliminate. From ANC-ON shown in Figure 20, it can be seen that the signal amplitude of the noise at the outlet of the PVC pipe can be reduced by about $17 \mathrm{~dB}$ after a very short time, indicating that the proposed method has a practical effect of noise reduction. It can also be seen from the 


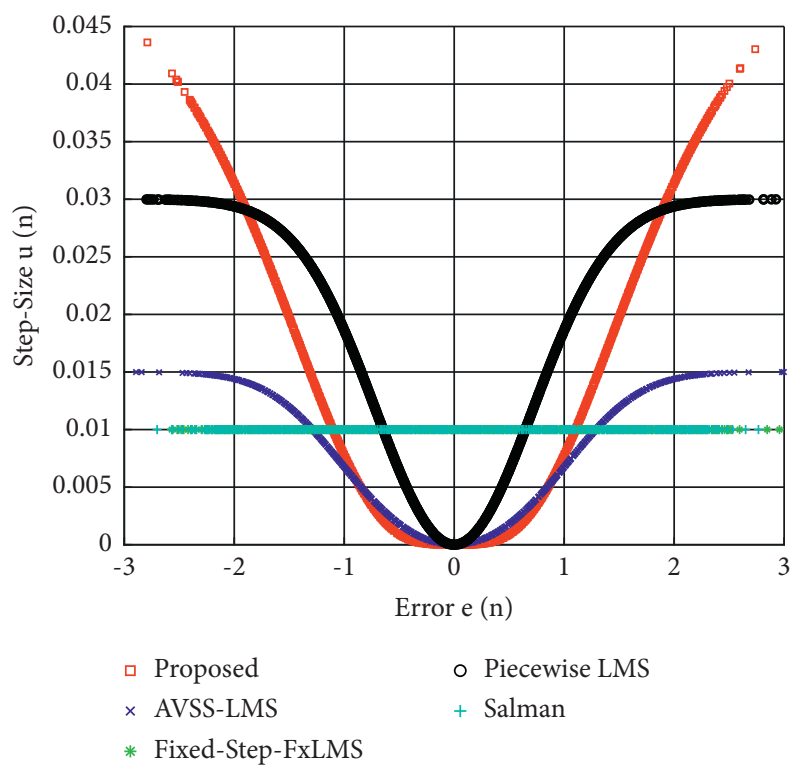

Figure 18: Comparison of step size of five noise reduction methods.

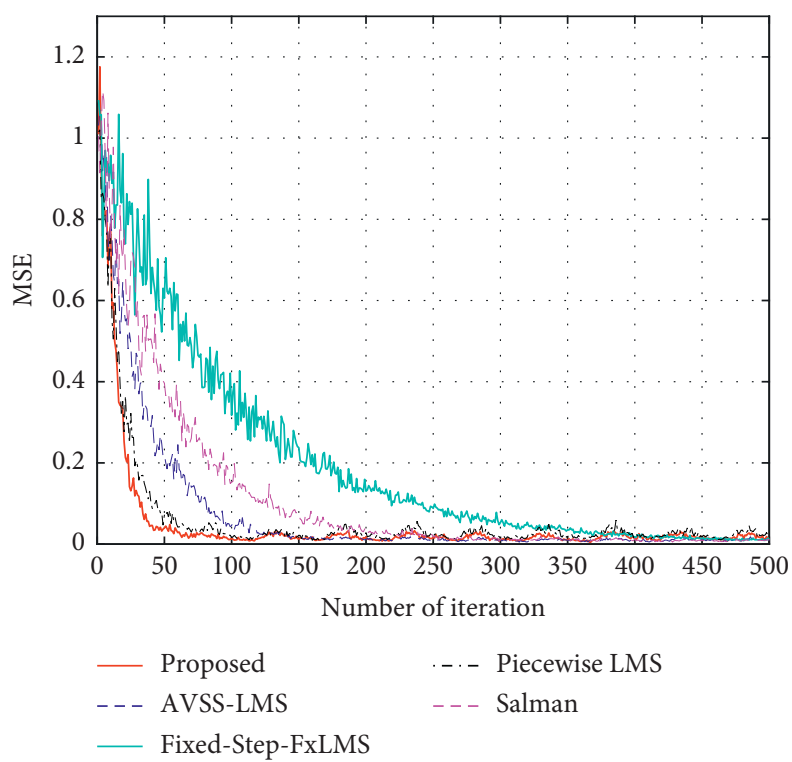

FIGURE 19: Comparison of MSE of five noise reduction methods.

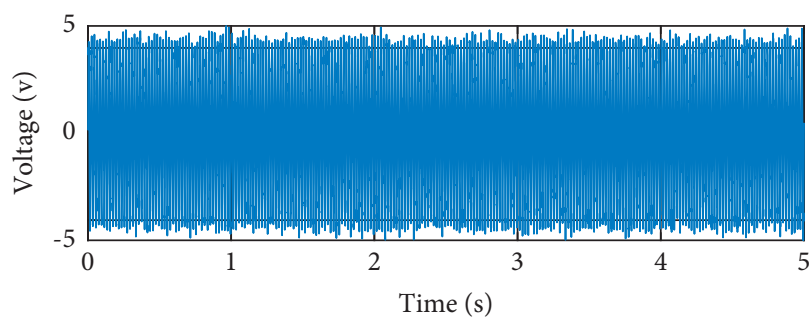

(a)

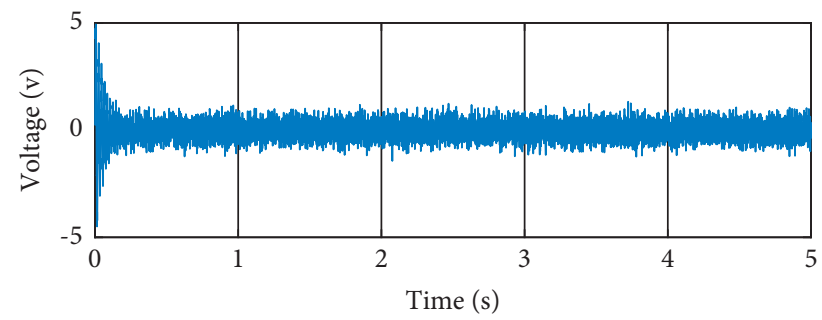

(b)

FIgure 20: Comparison of (a) ANC-OFF and (b) ANC-ON. 
TABle 4: Performance comparison.

\begin{tabular}{lcccc}
\hline \multirow{2}{*}{ Method } & & \multicolumn{2}{c}{ Comparison parameters } \\
& Maximum step size & Number of iterations & Noise cancellation effect (dB) & Convergence time (ms) \\
\hline Proposed & 0.042 & 41 & 17.2 & 4.05 \\
AVSS-LMS & 0.015 & 70 & 16.0 & 5.91 \\
Salman & 0.01 & 220 & 15.5 & 18.21 \\
FXLMS & 0.01 & 413 & 14.3 & 22.3 \\
Piecewise & 0.03 & 55 & 14.9 & 4.25 \\
\hline
\end{tabular}

experimental results that the improved method has a more satisfactory effect on the regular noise cancellation, while there is still some room for improvement for random noise.

In Table 4, we compare the maximum step size that can be achieved by the five algorithms, the number of iterations when $\mathrm{MSE} \leq 0.1$, and the noise cancellation effect when applied to pipelines. The test environment for the five algorithms is consistent with the test signal. The proposed, AVSS-LMS, piecewise, fixed-step FXLMS, and Salman algorithms take $0.9901,0.9438,0.7719,0.5406$, and $0.8281 \mathrm{~s}$, respectively, to perform 10000 iterations. In Table 4, the convergence time is the product of the average single-step iteration time and the number of iterations. Although the total time for the proposed algorithm to complete $n=10,000$ iterations is the longest, it takes only a few iterations to converge, so the time for the proposed algorithm that converges to MSE $\leq 0.1$ is shorter than that of the other four algorithms. It can be seen that the maximum step size that can be obtained by the proposed method is larger compared to the other four algorithms, and therefore, the number of iterations is relatively less. In addition, the comparison in Table 4 also provides that the improved method can reduce more noise than the remaining four methods, and AVSS-LMS also has a better noise cancellation effect. The piecewise method is influenced by the segmentation strategy, and the segmentation points need to be retested after the usage environment has changed.

The proposed algorithm can quickly eliminate noise in pipelines. It has good tracking performance for rotation noise. The piecewise LMS algorithm can also converge rapidly for pipeline noise, and at the beginning of the iteration, it can achieve convergence performance close to that of the proposed algorithm (Figure 18). However, Figure 19 shows that MSE of the piecewise LMS algorithm is significantly higher than that of the proposed algorithm at a stable state, which is attributed to the nonsmooth change in the step size of the piecewise LMS algorithm at a steady condition.

Based on these results, compared with the other four algorithms, the proposed algorithm has a better suppression effect on rotation noise generated by the axial fan in HVAC pipelines. The algorithm uses the arctangent function to nonlinear the error signal and step size. In the effective noise reduction area, the random noise can be reduced by $12-17 \mathrm{~dB}$, and the noise is reduced by about $83 \%-92 \%$. Experiments and simulations show that the proposed algorithm has advantages in terms of convergence speed, steady-state error, and noise cancellation effect, and its comprehensive performance is better than that of the other four algorithms.

\section{Conclusion}

This study proposes a new variable step size FXLMS algorithm for ANC systems in HVAC pipelines. We present a variable step size FXLMS method based on the arctangent function based on in-depth evaluations of Neil, Wang, and Salman's step size modification strategies. The algorithm uses the boundedness and continuity of the arctangent function to nonlinearly transform the error signal, so that the step size can be adjusted following the change in the error signal, compared with the mainstream ANC algorithms that significantly improved convergence speed. It reduces the noise generated by the axial flow fan in HVAC systems and has a good noise cancellation effect. The proposed algorithm has an excellent response on standard PVC pipelines, but the algorithm may not converge for nonstandard pipelines due to the mismatch of the air pipeline $\mathrm{SP}$. As a result, the algorithm may not converge when used for nonstandard PVC pipelines. A further research task in the future is to investigate how to provide a more accurate SP model for the algorithm, so that our proposed algorithm can be easily used in various types of HVAC systems.

\section{Abbreviations}

ANC: $\quad$ Active noise control

HVAC: Heating, ventilation, and air-conditioning

VSS-LMS: Variable step size least mean square

FXLMS: $\quad$ Filtered-X least mean square

MSE: $\quad$ Mean square error

SP: $\quad$ Secondary path

SVS-LMS: $\quad$ Sigmoid variable step size LMS

AVSS-LMS: Antiinterference variable step size LMS

TVOCs: Total volatile organic compounds

PVC: $\quad$ Polyvinyl chloride

SNR: $\quad$ Signal noise ratio.

\section{Data Availability}

The data used to support the findings of this study are included within the article.

\section{Conflicts of Interest}

The authors declare that they have no conflicts of interest.

\section{Acknowledgments}

This study was supported by the National Natural Science Foundation of China (61871318). 


\section{References}

[1] S. Seyam, "Types of HVAC systems." HVAC System, IntechOpen, London, UK, 2018.

[2] A. Kar, A. Anand, and B. Majhi, "An improved variable-step FXLMS for active noise control in high-noise environment," in Proceedings of the TENCON 2019 - 2019 IEEE Region 10 Conference (TENCON), pp. 332-337, Kochi, India, October 2019.

[3] T. Padhi, M. Chandra, A. Kar, and M. N. S. Swamy, "A new adaptive control strategy for hybrid narrowband active noise control systems in a multi-noise environment," Applied Acoustics, vol. 146, pp. 355-367, 2019.

[4] H. Meng and S. Chen, "Particle swarm optimization based novel adaptive step-size FxLMS algorithm with reference signal smoothing processor for feedforward active noise control systems," Applied Acoustics, vol. 174, Article ID 107796, 2020.

[5] D. R. Morgan, "History, applications, and subsequent development of the FXLMS Algorithm [DSP History]," IEEE Signal Processing Magazine, vol. 30, no. 3, pp. 172-176, 2013.

[6] I. T. Ardekani, H. Sharifzadeh, and S. Rehman, "Efficient fxlms algorithm with simplified secondary path models," in Proceedings of the IEEE International Conference on Acoustics, Speech and Signal Processing (ICASSP), pp. 609-613, South Brisbane, Australia, April 2015.

[7] Y. Zhou, Q. Zhang, and Y. Yin, "Active control of impulsive noise with symmetric $\alpha$-stable distribution based on an improved step-size normalized adaptive algorithm," Mechanical Systems and Signal Processing, vol. 56, pp. 320-339, 2015.

[8] C. Wang, L. He, and Y. Li, "An anti-interference variable-step adaptive algorithm and its application in active vibration control," in Proceedings of the Ocean Acoustics, January 2016.

[9] M. S. Salman, F. El-Sayed, and A. Youssef, "A sparse variable Step-Size LMS algorithm for impulsive noise," in Proceedings of the 3rd International Conference on Bio-engineering for Smart Technologies (BioSMART), pp. 1-4, Paris, France, April 2019.

[10] N. J. Bershad, C. José, and M. Bermudez, "A switched variable step size NLMS adaptive filter," Digital Signal Processing, vol. 101, 2020.

[11] W. Z. Shen, "Aeroacoustic modelling of low-speed flows," Theoretical and Computational Fluid Dynamics, vol. 13, pp. 271-289, 1999.

[12] H. Luan, L. Weng, and Y. Luan, "Numerical simulation of unsteady aerodynamic interactions of contra-rotating axial fan," PLoS One, vol. 13, Article ID e0200510, 2018.

[13] E. A. Manzano and E. J. L. Lescano, "Optimal step-size of delayed FXLMS algorithm for multi-frequency active noise control systems," in Proceedings of the International Conference on Information Systems and Computer Science (INCISCOS), pp. 203-207, Quito, Ecuador, November 2019.

[14] B. Farhang-Boroujeny, Adaptive Filters: Theory and Applications, John Wiley \& Sons, New Jersey, NY, USA, 2nd edition, 2013.

[15] S. Haykin, Adaptive Filter Theory, Prentice-Hall, Upper Saddle River, NY, USA, 5th edition, 2014.

[16] T. S. Park, D. W. Kim, and P. Park, "A filtered-x VSS-NSAF active noise control algorithm robust to impulsive noise through the application of step-size scaler," in Proceedings of the 15th International Conference on Electrical Engineering/ Electronics, Computer, Telecommunications and Information Technology (ECTI-CON), pp. 744-747, Chiang Rai, Thailand, July 2018.
[17] I. T. Ardekani, H. Sharifzadeh, S. Rehman, and W. H. Abdulla, "Efficient fxlms algorithm with simplified secondary path models," in Proceedings of the IEEE International Conference on Acoustics, Speech and Signal Processing (ICASSP), pp. 609-613, South Brisbane, Australia, April 2015.

[18] Q. Niu and T. Chen, "A new variable Step-Size LMS adaptive algorithm," in Proceedings of the Chinese Control And Decision Conference (CCDC), pp. 1-4, Shenyang, China, June 2018.

[19] S. Binbin, L. Xiafu, and Z. Shuang, "An improved LMS adaptive filtering algorithm and its analysis," in Proceedings of the International Conference on Intelligent Computing Automation and Systems (ICICAS), pp. 549-551, Chongqing, China, December 2019.

[20] H. Yiding, W. Fengsen, Y. Min, and Y. Jianyi, "An improved variable step size LMS adaptive filtering algorithm," Computer Simulation, vol. 37, pp. 291-295, 2020.

[21] M. Kai, W. Pingbo, and W. Cai, "A variable step size LMS algorithm based on normal distribution curve," Computer Simulation, vol. 36, pp. 295-299, 2019.

[22] Y. Wei and Z. Yan, "Variable tap-length LMS algorithm with variable tap-length adaptation Step-Size," in Proceedings of the Chinese Control and Decision Conference (CCDC), pp. 37483751, Yinchuan, China, May 2016.

[23] Y. Qian, "A new variable Step-Size algorithm applied in LMS adaptive signal processing," in Proceedings of the Chinese Control and Decision Conference (CCDC), pp. 4326-4329, Yinchuan, China, May 2016.

[24] B. Hassibi, A. H. Sayed, and T. Kailath, " $\mathrm{H}_{\infty}$ optimality of the LMS algorithm," IEEE Transactions on Signal Processing, vol. 44 , pp. 267-280, 1996.

[25] I. D. Landau and R. Meléndez, "Active noise control: adaptive vs. robust approach," in Proceedings of the 25th Mediterranean Conference on Control and Automation (MED), pp. 799-804, Valletta, July 2017.

[26] A. Haseeb, M. Tufail, S. Ahmed, and W. Ahmed, "A robust approach for online feedback path modeling in single-channel narrowband active noise control systems using two distinct variable step size methods," Applied Acoustics, vol. 133, pp. 133-143, 2018.

[27] M. Yaping, X. Yegui, and M. Liying, "Statistical analysis of narrowband active noise control using a simplified variable step-size FXLMS algorithm," Signal Processing, vol. 183, 2021.

[28] R. Fraanje and S. J. Elliott, "Verhaegen M. Robustness of the filtered-X LMS algorithm-part II: robustness enhancement by minimal regularization for norm bounded uncertainty," IEEE Transactions on Signal Processing, vol. 55, pp. 40384047, 2007.

[29] C. Boultifat, P. Loiseau, P. Chevrel, and J. Loheac, "FxLMS versus $\mathrm{H} \infty$ control for broadband acoustic noise attenuation in a cavity," IFAC-PapersOnLine, vol. 50, pp. 9204-9210, 2017.

[30] W. Z. Shen, "Aeroacoustic modelling of low-speed flows," Theoretical and Computational Fluid Dynamics, vol. 13, pp. 271-289, 1999.

[31] V. I. Tokarev, O. I. Zaporozhets, and V. M. Vorotyntsev, "Sound generation by airborne air conditioning systems: theory and analysis," Applied Acoustics, vol. 55, pp. 145-162, 1998.

[32] F. Farassat, "Linear acoustic formulas for calculation of rotating blade noise," AIAA Journal, vol. 19, pp. 1122-1130, 1981.

[33] S. F. Wu, S. G Su, and H. S. Shah, "Modelling of the noise spectra of axial flow fans in a free field," Journal of Sound and Vibration, vol. 200, pp. 379-399, 1997. 
[34] M. L. Munjal, Acoustics of Ducts and Mufflers 2nd Ed, WileyInterscience, New York, NY, USA, 2014.

[35] A. Jameson and L. Martinelli, "Optimum aerodynamic design using the Navier-Stokes formulas," Theoretical and Computational Fluid Dynamics, vol. 10, pp. 213-238, 1998.

[36] P. Drabek, "The regenerated noise of HVAC elements and its measurement in pipeline track," MATEC Web of Conferences. EDP Sciences, vol. 210, 2018.

[37] E. Envia, "Fan noise reduction: an overview," International Journal of Aeroacoustics, vol. 1, pp. 43-64, 2002.

[38] C. Ying, Z. Lai-bin, and W. Liang, "Study of noise reduction of natural gas pipeline leakage acoustic series by local projection," Journal of China University of Petroleum (Edition of Natural Science, vol. 1, pp. 118-124, 2010.

[39] L. Jing, Z. Li, Y. Li, and R. D. Murch, "Channel characterization of acoustic waveguides consisting of straight gas and water pipelines," IEEE Access, vol. 6, pp. 6807-6819, 2018.

[40] A. D. Pierce, Acoustics: An Introduction to its Physical Principles and Applications, Springer, Berlin, Germany, 2019.

[41] M. S. Kim, S. K. Lee, S. Y. Jang, and J. P. Koh, “Acoustic wave propagation characteristics corresponding to the cut-off frequency in gas pipeline," Transactions of the Korean Society for Noise and Vibration Engineering, vol. 18, pp. 693-700, 2008.

[42] M. Abdullahi and S. O. Oyadiji, "Acoustic wave propagation in air-filled pipes using finite element analysis," Applied Sciences, vol. 8, p. 1318, 2018.

[43] M. T. Akhtar, "On active impulsive noise control (AINC) systems," Circuits, Systems, and Signal Processing, vol. 39, pp. 4354-4377, 2020. 